NAG39702

\title{
Aging in lattice-gas models with constrained dynamics
}

\author{
Jorge Kurchan \\ École Normale Supérieure de Lyon, 46, Allée d'Italie, F-69364 Lyon Cedex 07, France. \\ Luca Peliti目 and Mauro Sellitto 3 \\ Dipartimento di Scienze Fisiche and Unità INFM, \\ Università "Federico II", Mostra d'Oltremare, Pad. 19, I-80125 Napoli, Italy.
}

(September 13, 2018)

\begin{abstract}
We investigate the aging behavior of lattice-gas models with constrained dynamics in which particle exchange with a reservoir is allowed. Such models provide a particularly simple interpretation of aging phenomena as a slow approach to criticality. They appear as the simplest three dimensional models exhibiting a glassy behavior similar to that of mean field (low temperature mode-coupling) models.
\end{abstract}

05.20.-y, 64.70.Pf, 61.20.Ja

Typeset using REVTEX 
As the temperature of a supercooled liquid is reduced, its dynamics becomes slower and slower and its viscosity increases. The glassy transition is usually located at the temperature $T_{g}$ where the viscosity first exceeds the conventional value of $10^{13}$ Poise [4]. At lower temperatures, the glass is usually assumed to be "solid" in the sense that its molecules rattle within a "cage" formed by their neighbors and do not move away from it, at least within experimental time scales. However the glassy state is not an equilibrium one since the evolution of the system does not stop altoghether, but rather keeps evolving at a slower and slower pace as the time $t_{w}$ elapsed since its quench increases. This is the origin of the striking aging effects observed in glasses [5:6]. Aging corresponds to the property that, while one-time quantities like the average energy, volume, etc., appear to be invariant under time translations, two-time correlations and responses exhibit a non trivial dependence on both of their arguments [7]. Similar properties have also been observed in spin-glasses [8]. These aging properties have been thoroughly investigated within spin-glass models [9] which are closely related to the mode-coupling theories of structural glasses [1]. Aging appears provided one takes the infinite size limit before taking the infinite time limit [10]. In this situation, the Boltzmann-Gibbs equilibrium distribution is never reached. Conventional mode-coupling theories, which are based on the hypothesis that the initial distribution is the equilibrium one, are therefore inconsistent in this limit below $T_{g}$.

The spin-glass models proposed as a description of structural glasses lack, however, a transparent physical interpretation in terms of particles and involve a complex, random hamiltonian which is hard to justify as a description of a fluid. Their justification is rather a posteriori in the sense that the phenomena they exhibit recall the behavior of structural glasses. On the other hand a class of very simple kinetic models have been introduced to describe the slowing down of the dynamics [11, 12]. These models are defined by kinetic rules involving a selection of the possible configuration changes ("moves") and are therefore called models with constrained dynamics. The kinetic rules satisfy detailed balance and are compatible with a Boltzmann-Gibbs equilibrium distribution involving a hamiltonian, usually chosen to be a trivial one. The constraints are alone responsible for the slowing down 
of the dynamics because, near any allowed configuration, there are only few configurations which satisfy them.

Some out of equilibrium properties in models of this kind have been already discussed in [13]. We shall in this paper refer to the kinetic lattice-gas model studied by Kob and Andersen (KA) [14]. The system consists of $N$ particles in a cubic lattice of side $L,\left(V=L^{3}\right)$ with periodic boundary conditions. There can be at most one particle per site. At each time step a particle and one of its neighbouring sites are chosen at random. The particle is moved if the three following conditions are all met: (i) the neighbouring site is empty; (ii) the particle has less than four nearest neighbours; (iii) the particle will have less than four nearest neighbours after it is moved. The rule is symmetric in time, detailed balance is satisfied and the allowed configurations have equal probability in equilibrium. In this model the dynamics becomes slower and slower as the particle density $\rho$ increases. Above a critical value of the density, $\rho_{c}$, particle diffusion stops completely. These investigations are carried on initializing the system in an "equilibrium" configuration: therefore the approach cannot be extended below the glass transition temperature without becoming vulnerable to the same objection voiced above against the mode coupling theories: how can one reach this "equilibrium" configuration?

We show in this paper, by numerical simulation, that it is possible to study aging in these models, if they are provided with a mechanism allowing one to realize a process analog to a quench. We consider the three-dimensional KA model, but we allow the lattice gas to exchange particles on a single two-dimensional layer with a reservoir characterized by the chemical potential $\mu$ [15]. For each value of $\mu$ it is trivial to evaluate the equilibrium value of the density $\rho_{e q}(\mu)$. If $\rho_{e q}(\mu)<\rho_{c}$, the system rapidly reaches the equilibrium state. There is therefore a critical value, $\mu_{c}$, of $\mu$, defined by $\rho_{e q}\left(\mu_{c}\right)=\rho_{c}$. A quenching process corresponds to performing a jump of $\mu$ from below to above $\mu_{c}$. Therefore, $\mu$ plays a role analogous to the inverse temperature in mode-coupling theories. We then observe that $\rho$ never exceeds $\rho_{c}$, but rather approaches it like a power law in time, and that the square displacement and the self-correlation function of the particle violate time-translation invariance, since they 
explicitly depend on the waiting time, $t_{w}$, after the quench.

One can also perform "annealing" (compression) experiments, where $\mu$ is increased at a fixed rate. In close resemblance with the behavior of real glasses, we observe that the limit density depends on the compression rate, and tends to $\rho_{c}$ as the rate decreases to zero. We can then evaluate, by integration, the entropy variation of the reservoir. The curves remain consistently above the equilibrium curve $S=S_{e q}(\mu)$, and seem to reach a critical value, $S_{c}$, as the compression rate decreases to zero. This is reminiscent of the Kauzmann paradox, which is usually considered as an argument for the existence of a thermodynamical phase transition related to the glass one. However in this case, we know that there is no such transition.

Hysteresis effects also appear when the reservoir's chemical potential is varied cyclically.

As mentioned in the Introduction, KA studied the diffusion and the decay of the correlation functions evolving at fixed number of particles starting from a random (equilibrium) configuration. They found that correlations decayed to zero with a characteristic time $\tau(\rho)$ that appears to diverge at $\rho_{c} \sim 0.881$, where also the diffusivity constant goes to zero. For densities above $\rho_{c}$, the correlations did not decay at all.

We modify the KA model by creating and destroying particles on a single slice, say $(x, y, z)=(1, y, z)$ (the "surface") with the following Montecarlo rule: We choose a site on that slice at random. If it is empty we add a particle, if it is occupied we remove the particle with probability $\mathrm{e}^{-\mu}$. We alternate such sweeps of creation/destruction with the ordinary diffusion sweeps. Since the creation/destruction mechanism is very fast, the surface itself is always in equilibrium.

It is easy to calculate the thermodynamical equilibrium quantities (we set the temperature $k_{B} T=1$ throughout): $1 / \rho=1+\mathrm{e}^{-\mu}$, where $\rho$ is the density. The entropy per unit volume is given by $S=-\rho \ln \rho-(1-\rho) \ln (1-\rho)$.

We first consider compression experiments in which $1 / \mu$ (a quantity analogous to temperature) is slowly lowered from a high value to zero. In Fig. Tla we report the results of the specific volume $v=1 / \rho$ versus $1 / \mu$ for several annealing speeds. The smooth curve is the 
equilibrium state equation and the horizontal dashed line is the critical specific volume of $\mathrm{KA} v_{c}=1 / \rho_{c}=1 / 0.881$. In Fig. 四, we show the change in entropy of the reservoir, given by $S(\mu)=S\left(\mu_{i}\right)-\int_{\mu_{i}}^{\mu} \mu d \rho$. The smooth curve is the equilibrium curve $S$ vs. $1 / \mu$, and the horizontal dashed line is the entropy at the critical density. These curves look quite similar to the energy vs. temperature and entropy versus temperature curves in the annealing of glasses. As predicted, the system falls off equilibrium around $\mu_{c}$. We have checked that the bulk of the sample does not exhibit density inhomogeneities except very close to the "surface".

We now turn to the behaviour of the system after a quench to the subcritical value $1 / \mu<1 / \mu_{c}$. Here we concentrate on the behaviour of a large system at long but finite times. In Fig. 2 we plot the specific volume minus its asymptotic value versus time after a quench to the subcritical value $1 / \mu=1 / 2.2$. The data can be fitted by a power law of the form $v(t)-v_{\infty} \sim t^{-\alpha}$, where $\alpha=0.57 \pm 0.02$. The asymptotic value of the density $\rho_{\infty}=1 / v_{\infty}=0.861$ is slightly lower than the KA value $\rho_{c}=0.881$, suggesting that the critical density might have a weak dependence on the way the particles entered the bulk (although it might also be a finite-size effect).

The mean square displacement $R^{2}\left(t+t_{w}, t_{w}\right)$ of a particle between time $t_{w}$ and time $t+t_{w}$ must be defined with some care, since the particles may leave or enter the system. We define it by averaging only over the particles which are present at both times. We find that diffusion indeed slows down as the waiting time increases. However, the time $t\left(R^{2}\right)$ needed to reach a given square distance is roughly proportional to $t_{w}$ only for long times. In fact, we find here a phenomenon already encountered by Kob and Barrat [17] in Lennard-Jones systems: one has to consider an effective waiting time that takes into account the relaxation of the system that happened already before the quench. Hence, one should define an effective waiting time $t_{w}^{e f f}=t_{w}+\tau_{0}$, where $\tau_{0}$ is the relaxation time characteristic of the equilibrium situation before the quench. Fig. 3 shows that, if one plots the squared displacement vs. $t / t_{w}^{e f f}$, the curves lie roughly on top of each other. The deviations are probably due to finite size effects, e.g. to particles that escape from the sample. The plot exhibits the waiting-time 
dependence of the diffusion constant and is consistent with a simple argument suggesting that diffusion is logarithmic in the presence of aging [18].

One can also compute easily the effective potential of Franz and Parisi [16]. This potential is a purely static quantity, and therefore is independent of the dynamical constraints. Indeed, we have not found any signature for the dynamical transition in the effective potential. However the "number of neighbours" constraint is an idealization of microscopical (repulsive) energy terms, and as such it should be seen by the effective potential. It would be interesting to clarify this point further.

Since the size of the system considered here is finite, equilibrium must eventually be reached, provided that any two allowed configurations can be connected by a path of allowed moves. It is possible to convince oneself that the kinetic rules allow an initially empty lattice to be progressively filled in, leaving only $\mathrm{O}(1 / L)$ empty sites per unit volume, and that it is possible to find a path connecting almost any two allowed configurations, if necessary by letting the particles escape one by one by the way they got in. Therefore, for any value of $1 / \mu$, the equilibrium density will eventually be reached, but with times which diverge fast as $L \rightarrow \infty$. We have verified that a system of size $5^{3}$ equilibrates after a time of order $10^{8}$ MC sweeps. What this argument implies is that after a quench there is first the power-law approach to a critical density $\rho_{c}$, followed by a much slower (size-dependent) increase in density to the equilibrium density.

We have thus shown that the out of equilibrium dynamics of kinetic lattice-gas models reproduce the phenomenology of more sophisticated glass models if one endows them with a surface mechanism of particle exchange with a reservoir. Equivalently, we could have coupled the system to a piston and used the pressure as an external parameter instead of the reservoir's chemical potential. The qualitative behaviour of this model is similar to that of mean-field spin-glass models, whose dynamical equations are exactly (in the infinite-size limit) the "idealised" mode-coupling equations and their correct low-temperature extension. The roles played by the inverse chemical potential and the inverse density (specific volume) in the present model are analogous to the ones played by temperature and energy 
respectively, in the mean-field case. When quenched below $T_{c}$, mean-field spin-glass models never equilibrate. Their energy relaxes to a "threshold" value $E_{\text {thres }}(T)$ which is higher than the equilibrium one [10], and can be characterized as the value below which the phase space breaks into disconnected components. In our case, the moves allowed by the kinetic constraints keep the phase space of the system connected only for $\rho<\rho_{c}$. Therefore the density approaches this threshold value, and the corresponding manifold of allowed states approaches to the incipient "percolation" cluster characteristic of $\rho_{c}$ [20]. Only for finite sizes the system is able to reach values of $\rho$ larger than $\rho_{c}$, just as energy values below the threshold can only be reached by finite systems in mean-field spin-glasses [10,19.

In short: in both cases the system ages because it slowly evolves towards a situation in which the available phase-space becomes completely disconnected [20]. The closer the system to the "phase-space percolation" threshold level, the slower its dynamics becomes. In this sense the system ages because it approaches criticality [21].

To summarize, lattice gas models with constrained dynamics, in spite of their simplicity, capture the essential features of the aging behavior of three dimensional glasses.

We are indebted to J.-L. Barrat for suggesting to look at the effective potential and to J.-P. Bouchaud, L. Cugliandolo, P. Holdsworth and W. Kob for useful discussions and suggestions. M.S. acknowledges the hospitality of the Laboratoire de Physique Théorique of École Normale Supérieure de Lyon, where part of this work was performed. 


\section{REFERENCES}

[1] E-mail address: Jorge.Kurchan@enslapp.ens-lyon.fr

[2] Associato INFN Sezione di Napoli. E-mail addresses: luca@turner.pct.espci.fr, peliti@na.infn.it

[3] E-mail address: Mauro.Sellitto@na.infn.it

[4] See e.g.: W. Götze, L. Sjögren, Rep. Prog. Phys. 55, 241 (1992).

[5] L.C.E. Struik, Physical Aging in Amorphous Polymers and Other Materials, (Houston: Elsevier, 1978).

[6] I. Hodge, J. Non Cryst. Solids 169, 211 (1994); Science 267, 1945 (1996).

[7] For a recent review, see: E. Vincent, J. Hammann, M. Ocio, J.-P. Bouchaud and L.F. Cugliandolo, in Proceeding of the Sitges Conference on glassy systems, June 1996, edited by E. Rubí, cond-mat $\mathbf{9 6 0 7 2 2 4 .}$

[8] L. Lundgren, P. Svedlindh, P. Nordblad and O. Beckman, Phys. Rev. Lett. 51911 (1983); E. Vincent, J. Hammann and M. Ocio; in Recent Progress in Random Magnets, edited by D. H. Ryan, (Singapore: World Scientific, 1992) p. 207.

[9] J.-P. Bouchaud, L.F. Cugliandolo, J. Kurchan and M. Mézard, cond-mat 9702070, and references therein; for a review on aging simulations in spin-glasses see: H. Rieger, Ann. Rev. of Comp. Phys. II, ed. D. Stauffer (World Scientific, Singapore, 1995).

[10] L.F. Cugliandolo and J. Kurchan, Phys. Rev. Lett. 71, 173 (1993); Phil. Magaz. B 71, $50(1995)$.

[11] G.H. Fredrickson and H.C. Andersen, Phys. Rev. Lett. 53, 1244 (1984); J. Chem. Phys. 83, 5822 (1985); G.H. Fredrickson and S.A. Brawer, J. Chem. Phys. 84, 3351 (1986).

[12] W. Ertel, K. Froböse and J. Jäckle, J. Chem. Phys. 88, 5027 (1988). K. Froböse, J. Stat. Phys. 55, 1285 (1989). J. Jäckle, K. Froböse and D. Knölder, J. Stat. Phys. 63, 
249 (1991). J. Reiter, F. Mauch and J. Jäckle, Physica A 184, 458 (1992). J. Jäckle and D. Sappelt, Physica A 192, 691 (1993).

[13] E. Follana and F. Ritort, Phys. Rev. B 54, 930 (1996). See also a density functional lattice model in: F.G. Padilla and F. Ritort, cond-mat 9703095 (1997), where creation and destruction of particles within the bulk is allowed.

[14] W. Kob and H.C. Andersen, Phys. Rev. E 48, 4364 (1993).

[15] Coupling a kinetic lattice-gas model with a particle reservoir has been considered by A. Imparato, University of Naples thesis, unpublished. D. Stariolo, cond-mat 9612082, has suggested that aging may be a consequence of mass non-conservation in diffusion models.

[16] S. Franz and G. Parisi, J. Phys. (France) I 5, 1401 (1995).

[17] W. Kob and J.-L. Barrat, cond-mat 9704006.

[18] G. Parisi, cond-mat 9703219.

[19] A. Barrat, R. Burioni and M. Mézard, J. Phys. A 29, L81 (1996).

[20] For discussions of this kind, see I.A. Campbell, J. Phys. Lett. 46, L1159 (1985); Phys. Rev. B 33, 3587 (1986). C.M. Newman and D.L. Stein, Phys. Rev. Lett. 72, 2286 (1994); D.L. Stein and C.M. Newman, Phys. Rev. E 51, 5228 (1995).

[21] The connection between aging and self-organized criticality has been explored by: S. Boettcher and M. Paczuski, cond-mat 9702054; see also C. Tang and P. Bak, Phys. Rev. Lett. 60, 2347 (1988). We thank P. Bak for have pointed out to us these references. 


\section{FIGURES}
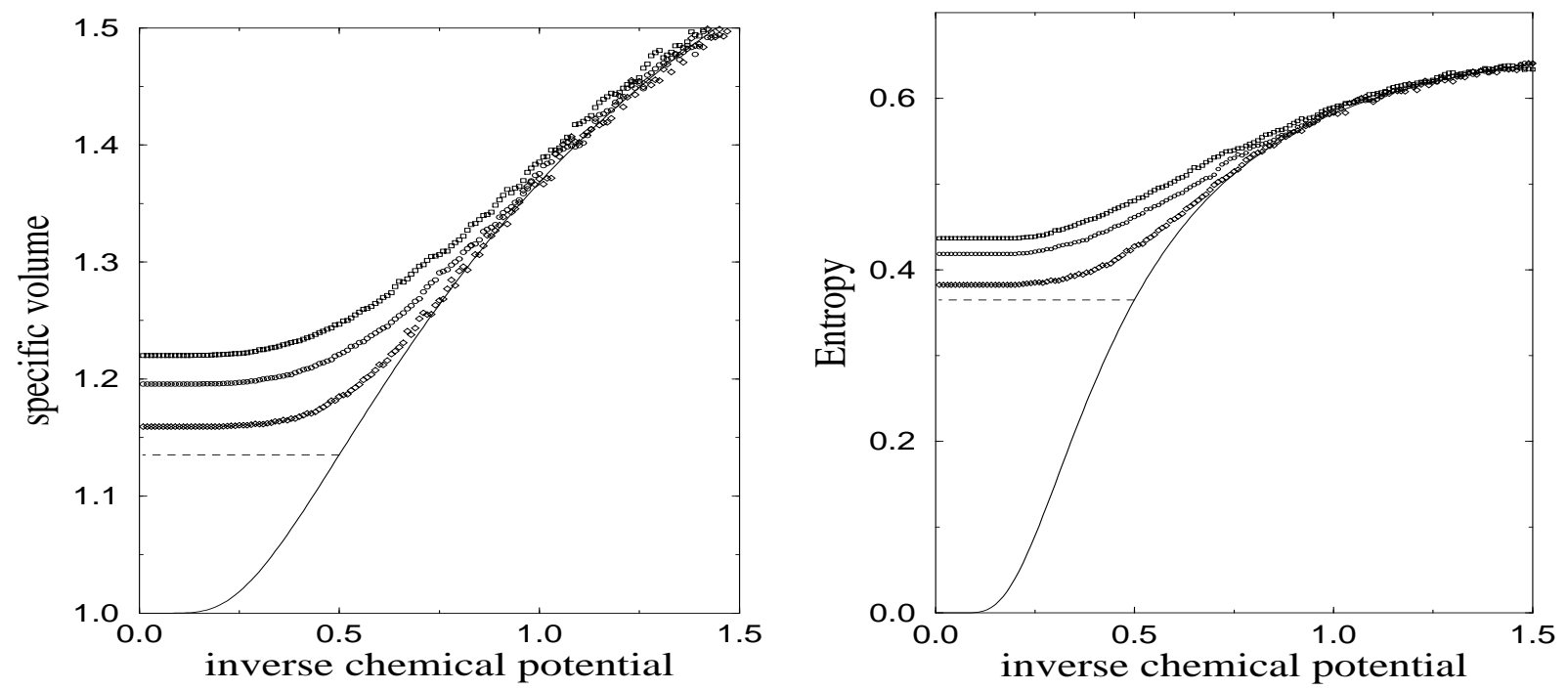

FIG. 1. a: Compression experiment. The smooth curve is the equilibrium state equation; while the dashed line is the KA critical value of specific volume. b: 'Entropy' as obtained by integration of compression experiment data. The smooth curve is the equilibrium entropy. The dashed line is the KA critical value of entropy. The compression rates are (from top to bottom) $3 \cdot 10^{4}, 10^{5}, 10^{6}$ sweeps per unit of inverse $\mu$. System of size $20^{3}$, averages over five samples.



FIG. 2. Relaxation of the excess specific volume $\Delta v=v(t)-v_{\infty}$ after a quench to the subcritical value $1 / \mu=1 / 2.2$. The straight line corresponds to $\Delta v \propto t^{-0.57}$. 


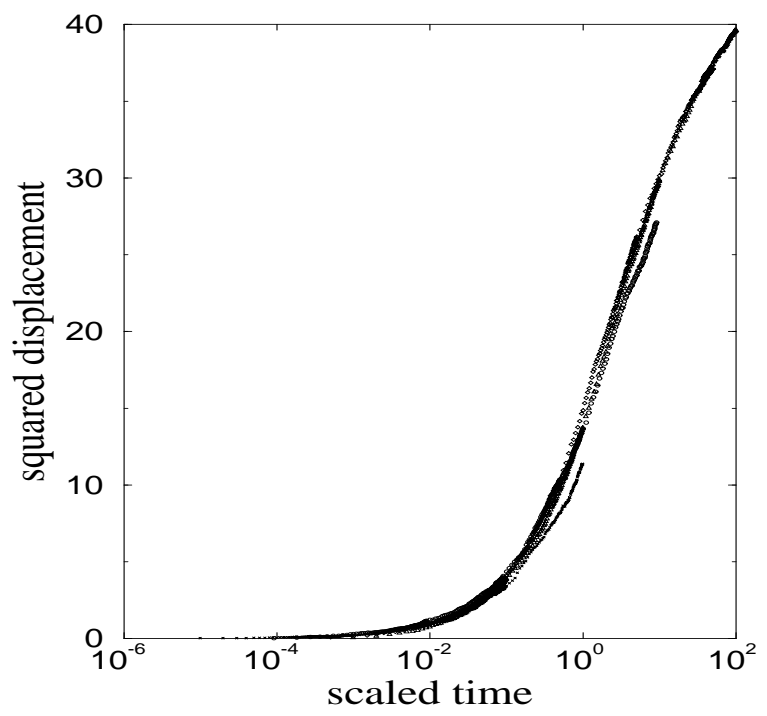

FIG. 3. Squared displacement $R^{2}\left(t+t_{w}, t_{w}\right)$ vs. scaled time $t / t_{w}^{e f f}$ for $t_{w}=10,10^{3}, 10^{4}, 10^{5}$, and $t_{0}=10^{3}\left(t_{w}^{e f f}=t_{w}+t_{0}\right)$. System of size $20^{3}$, average over five samples. 